Revue d'histoire de l'Amérique française

FEV REVUE D.HISTOIRE DE L'AMÉRIQUE FRANÇAISE

\title{
Premières réactions des vaincus de 1760 devant leurs vainqueurs
}

\section{Michel Brunet}

Volume 6, numéro 4, mars 1953

URI : https://id.erudit.org/iderudit/301550ar

DOI : https://doi.org/10.7202/301550ar

Aller au sommaire du numéro

Éditeur(s)

Institut d'histoire de l'Amérique française

ISSN

0035-2357 (imprimé)

1492-1383 (numérique)

Découvrir la revue

Citer cet article

Brunet, M. (1953). Premières réactions des vaincus de 1760 devant leurs vainqueurs. Revue d'histoire de l'Amérique française, 6(4), 506-516.

https://doi.org/10.7202/301550ar d'utilisation que vous pouvez consulter en ligne.

https://apropos.erudit.org/fr/usagers/politique-dutilisation/ 


\section{PREMIERES RÉACTIONS DES VAINCUS DE 1760 DEVANT LEURS VAINQUEURS}

Tous les historiens ont souligné les relations très cordiales qui s'établirent entre les Canadiens et leurs conquérants dès les premiers mois de l'occupation anglaise. Le fait demeure indéniable. On ne saura jamais exactement ce que les gens pensaient dans leur for intérieur mais leur attitude extérieure manifeste une entière soumission.

L'empressement des vaincus à se soumettre à l'autorité d'un ennemi séculaire, leur docilité, la servilité de leurs classes dirigeantes surprennent quelque peu un observateur du XXe siècle. Celui-ci a été témoin de la résistance courageuse des pays occupés par les Allemands au cours de la dernière guerre. Les mots "collaboration" et "collaborateurs" ont très mauvaise presse de nos jours. Nos manuels d'histoire contemporaine nous ont appris à admirer les luttes désespérées des minorités - Grecs, Belges, Polonais, Italiens, Hongrois - soumises à une domination étrangère. Il faut reconnấtre que la conduite des Canadiens, au moment de la conquête, n'a jamais inspiré et n'inspirera jamais les poètes à la recherche d'actes d'hérolsme collectif.

Comment expliquer la soumission presque spontanée des vaincus de 1760 ? L'historien A.L. Burt soutient que la bienveillance des militaires anglais, la totalité de leur victoire, l'impossibilité pour les Canadiens de songer à une revanche, les bienfaits que valut au pays le rétablissement de la paix contribuèrent à établir solidement et pacifiquement la domination anglaise au Canada ${ }^{1}$. Lyrique, il conclut: "De toutes les glorieuses victoires remportées par les armées britanniques, nulle n'est plus glorieuse, nulle n'est plus grande que la conquête morale qui vint couronner la conquête militaire du Canada2". L'explication de M. Burt ne tient pas suffisamment comp-

1. A.L. Burt, The Old Province of Quebec (Toronto, 1933), 55-56.

2. Ibid., 56 . 
te des vaincus eux-mêmes. Le même reproche pourrait s'adresser aux explications données par la plupart des autres historiens.

On n'a pas encore tenté d'écrire l'histoire de cette "conquête morale". Beaucoup plus grave que la conquête militaire dont elle n'est que la conśéquence, elle a complètement modifié l'évolution historique du peuple que la France avait établi sur les rives du SaintLaurent. Commencée en 1760 , cette "conquête morale" se continue depuis cette date. En retracer les étapes c'est décrire comment un peuple conquis perd le privilège de se développer normalement. $M$. Burt et tous ceux qui ont employé l'expression "conquête morale" ne se sont jamais arrêtés à en évaluer le sens terrible.

Dans le cadre limité de cet article, nous nous bornerons à rappeler les premières réactions des vaincus. Celles-ci furent la manifestation extérieure d'un état d'être que nous devons chercher à expliquer dans le contexte historique du moment où ils vivaient.

\section{Découragement des vaincus}

Les Canadiens vaincus s'attendaient au pire. La lutte avait été longue et sans merci. La propagande officielle n'avait cessé de leur répéter que l'Anglais vainqueur serait sans pitié. Rappelant le sort pénible des Acadiens, Mgr de Pontbriand avait mis ses ouailles en garde contre des ennemis qui "agissent d'une manière si irrégulière, et sur la parole desquels la prudence ne permet pas de se reposer". Il avait précisé:

Ainsi quand même ils consentiraient à vous laisser dans vos biens, quand même ils ne voudraient pas vous obliger a prendre les armes contre la France (ce qu'ils exigent des Acadiens), quand même ils permettraient la liberté de la Religion; ce qui vient de se passer dans l'Acadie rendrait suspectes toutes ces promesses, et vous auriez bientôt la douleur de voir s'introduire dans ce diocèse, dont la foi a toujours été si pure, les erreurs détestables de Luther et de Calvin. Vous allez done combattre dans cette année, non seulement pour vos biens, mais encore pour préserver ces vastes contrées de l'hérésie et des monstres d'iniquité qu'elle enfante à chaque moment ${ }^{3}$.

3. "Mandement pour des prières publiques - Dispersion des Acadiens", 15 février 1756, Mandements, lettres pastorales et circulaires des evếques de Québec, 2: 106107. 
L'ennemi lui-même n'avait pas ménagé ses menaces. La proclamation de Wolfe est justement demeurée célèbre: "Si, au contraire, un entêtement déplacé et une valeur imprudente leur fait prendre les armes, qu'ils [les Canadiens] s'attendent à souffrir tout ce que la guerre offre de plus cruel, s'il leur est aisé de se représenter à quel excès se porte la fureur d'un soldat effrénét". En remontant le fleuve Murray promit qu'une "vengeance sanglante punira ceux qui oseront avoir recours aux armes. Le ravage de leurs terres, l'incendie de leurs maisons seront les moindres de leurs malheurs." Le conquérant prouva qu'il était capable de passer des paroles aux actes. Les paroisses de la région de Québec connurent, au cours de l'été de 1759, la "fureur d'un soldat effréné". Quant à Murray, il démontra aux habitants des îles de Sorel et des environs qu'il pratiquait, lui aussi, l'art de la guerre totale.

La conduite des officiers français et des autorités métropolitaines rendit les vaincus encore plus malheureux. Le 15 juin 1760, Vaudreuil et Bigot leur annoncèrent que le gouvernement suspendait le paiement des lettres de change ${ }^{6}$. La propagande anglaise s'empressa d'expliquer aux Canadiens qu'ils étaient les victimes d'une banqueroute vraiment royale ${ }^{7}$. Ils s'y attendaient depuis longtemps. Bourlamaque parcourut la campagne montréalaise à la recherche des déserteurs. Il eut recours à l'incendie et au pillage pour convaincre les miliciens de faire leur devoir8.

Les Canadiens vécurent de 1759 à 1760 une année de cauchemar. Le vainqueur avait devant lui un vaincu prostré.

\section{Attitude bienveillante des vainqueurs}

Les Britanniques surent tirer parti des circonstances. L'état de prostration des vaincus servit admirablement les fins du conqué-

4. Proclamation de Wolfe, 27 juin 1759, dans Guy Frégault, François Bigot, administrateur français (2 v., Montréal, 1948), 2: 287.

5. Proclamation de Murray, 14 juillet 1760, dans "Proclamations du régime militaire", Rapport des Archives Publiques pour l'année 1918, 13. Cité à l'avenir sous le sigle RAP (1918).

6. Frégault, Bigot, 2: 319-320.

7. Proclamation de Murray, 27 juin 1760, RAP (1918), 11.

8. Frégault, Bigot, 2: 326-327. 
rant. Celui-ci se donna le beau rôle en se montrant généreux et compatissant. A peine entré dans Québec, Murray explique à son supérieur les multiples avantages d'une politique de clémence? $\mathrm{Pa}-$ ternel et protecteur, il calme les craintes d'une population résigné a tout:

Dans ces premiers moments de confusion, on n'a pu mettre tout l'ordre aux affaires qu'il y aurait voulu apporter, et nous ne sommes que trop persuadé qu'on a fait du tort à bien des pauvres habitants - Mais à cette heure que nous sommes plus arrangés. Nous avons résolu de maintenir une discipline exacte et rigoureuse, et de punir avec la dernière sévérité tous ceux qui seront convaincus de leur avoir fait la moindre injure ${ }^{10}$.

Murray se révèle un administrateur prévoyant et dévoué aux intérêts de la majorité. Pour remédier à la pénurie des moyens d'échange, il lance un emprunt ${ }^{11}$. Plus tard, il fixe le prix de la viande et du pain ${ }^{12}$. Au cours de l'hiver 1760-1761, officiers et soldats anglais de Québec secourent les pauvres de la capitale ${ }^{13}$. Le chanoine Briand, vicaire général dans le gouvernement de Québec, demande aux curés d'envoyer au gouverneur la liste des familles dans le besoin et d'“annoncer à vos peuples l'étroite obligation que leur imposent la piété et la reconnaissance de former des vœux pour Son Excellence, Monsieur notre Gouverneur, le charitable et généreux bienfaiteur 14". La sollicitude de Murray se manifeste tout particulièrement en faveur des religieuses hospitalières ${ }^{15}$. Pour mettre un frein à la spéculation et à l'agiotage, le gouverneur ordonne une déclaration des blés. Le clergé loue cette initiative humanitaire du conquérant et condamne l'avidité des paysans ${ }^{16}$.

9. Murray à Amherst, [ ?] novembre 1759, Papiers Murray, Archives Publiques du Canada (APC), M. 898 A.

10. "Mesures concernant la police pour Québec", 15 novembre 1759, RAP (1918), 3.

11. "Invitation à souscrire à l'emprunt public", 25 novembre 1759, ibid., 3.

12. Proclamation du 15 janvier 1760, ibid., 6.

13. Murray à Amherst, ler janvier 1761, Papiers Murray, APC, M. 898 D.

14. Lettre circulaire, Mandements, 2: 150.

15. Murray à Pitt, 3 décembre 1760, Papiers Murray, APC, M. 898 D.

16. "Circulaire au sujet d'une nouvelle déclaration des blés", 18 janvier 1761, Mandements, 2: 153-154. 
A Montréal et aux Trois-Rivières, les nouveaux maîtres se montrent des mieux disposés. Amherst avait assuré les habitants que les troupes paieraient leurs achats "en argent comptant et espèces sonnantes"17. Cette décision eut certainement le don de gagner au vainqueur une population pressurée par ses anciens dirigeants. Gage veille à l'approvisionnement régulier du marché montréalais et prend des mesures rigoureuses pour prévenir toute spéculation sur les vivres ${ }^{18}$. Il règle la vente du bois de chauffage dont le prix "s'est considérablement augmenté, au grand préjudice des pauvres, et causé seulement par l'avidité des propriétaires ${ }^{19}$ ". Les autorités interviennent pour faire diminuer le prix du pain ${ }^{20}$. Aux TroisRivières, le gouverneur Burton met en vigueur des règlements semblables à eeux adoptés par ses collègues de Québec et de Montréal ${ }^{21}$.

L'administration de la justice ne subit aucun changement radical. En ce domaine comme dans les autres, les autorités militaires procédèrent avec prudence et sagesse ${ }^{22}$. Les Canadiens n'eurent qu'à se louer de la situation ${ }^{23}$. Leurs craintes et les prophéties de malheur de la propagande française ne s'étaient pas matérialisées.

\section{Les vaincus reprennent espoir}

La générosité du conquérant, sa bienveillance, son souci de l'intérêt général, son esprit de justice lui acquirent le cœur des vaincus. Ceux-ci n'avaient qu'à dresser un parallèle entre la conduite des Anglais et celle des anciens administrateurs du pays. La comparaison, malheureusement, n'était pas a l'avantage de ces derniers. Un Canadien le déclara brutalement à un Français prêt à s'apitoyer sur l'avenir de la colonie: "Cessez, M[ onsieur ], d'avoir cette com-

17. Proclamation du gónéral Amherst, 22 septembre 1760, RAP (1918), 22.

18. "Règlements et ordres concernant la police générale", 26 octobre et 4 novembre 1760 , ibid., 33,34 .

19. "Ordonnance pour fixer le prix du bois d braler", 12 octobre 1761, ibid., 47.

20. "Ordonnance pour fixer le prix du pain", 18 octobre 1762, ibid., 61-62; "Ordonnance pour fixer le prix du pain et du ble", 9 novembre 1763, ibid., 77-78.

21. Voir ibid., 84s.

22. Voir proclamation d'Amherst, 22 septembre 1760, Documents constitutionnels, 1: 24-25.

23. Burt, Old Province, 32-35. 
passion pour nous, notre sort est moins malheureux qu'il n'étoit cydevant"24. Un correspondant français de M. de La Corne avait bien compris la situation: "Je pense que les Anglais, comme d'habiles gens, voudront traiter favorablement leurs nouveaux sujets pour se les attacher. Il leur en coûtera peu pour leur faire goûter l'avantage d'avoir changé de maîtres, puisqu'ils n'ont qu'à faire le contraire de ce que nous faisions"'25.

Des citoyens en vue de Montréal, se réclamant du Corps du Clergé, du Corps de Noblesse et du Corps du Commerce, s'empressent de faire leur cour au roi de Grande-Bretagne. Ils "osent prendre la liberté de se prosterner au pied de votre trône persuadés que c'est là où résident le sanctuaire de la justice, et le temple de toutes les autres vertus". Croyant qu'on a signé les préliminaires de paix, ils ne doutent pas qu'ils deviendront sujets de Sa Majesté Britannique et "c'est en cette qualité que nous avons recours au plus généreux et magnanime des rois. Tendre père de son peuple, nous nous flatterons qu'il daignera écouter le récit de nos infortunes"'26. En toute confiance, ils exposent leurs difficultés et sollicitent la permission d'importer de France. Ils espèrent se faire rembourser ainsi par le gouvernement français et s'engagent à limiter leurs importations au montant que celui-ci leur doit.

Burton remarque dans son rapport: "Les habitants, particulièrement les paysans, paraissent très satisfaits d'avoir changé de maîtres"27. Haldimand écrira à la fin de 1762, que "le commun bourgeois et les habitants, les seuls qu'il sera avantageux de garder avec le pays, sont fort tranquilles et paraissent attendre la décision de leur sort sans aucune inquiétude"28. Murray avait eu raison de

24. Hervieux, capitaine de milice, a un correspondant de France, 25 septembre 1761, dans Frégault, Bigot, $2: 334$.

25. Abbé de Frischeneau à M. de La Corne, 23 mars 1763, dans Auguste Gosselin, L'Eglise du Canada après la conquête (2 v., Québec, 1916-1917), 1: 64-65.

26. "Pétition des citoyens de la ville de Montréal a Sa Majesté Britannique", 12 février 1762, dans Documents relatifs d la monnaie, au change et aux finances du Canada sous le régime français (2 v., Ottawa, 1925), 2: 968.

27. Rapport de Burton, avril 1762, Documents constitutionnels, 1: 73.

28. Haldimand à Amherst, 26 décembre 1762, Collection Haldimand, APC, B 1: 262 . 
souligner que la conduite des Anglais étonnait les Canadiensa. Leurs chefs leur avaient annoncé qu'ils seraient persécutés, pillés, exterminés par le conquérant. Celui-ci, si on excepte quelques frictions inévitables, se montrait généreux et juste. Les leaders canadiens ne pouvaient que s'en réjouir.

M. Montgolfier, vicaire général, déclare aux Montréalais que le "général vainqueur de ce pays, aussi bien que celui qui a été établi pour nous gouverner, en entrant dans les vues du maítre commun, semblent n'avoir rien de plus à cœur que de faire disparaitre à nos yeux les horreurs de la guerre et de nous dérober en quelque façon jusques à la connaissance de la révolution, qui ne nous est demeurée sensible que par leur attention à procurer le bonheur et la tranquillité des peuples" 30 . Le chanoine Briand, fidèle aux conseils de Mgr de Pontbriand recommandant à ses prêtres de faire à l'occupant "toutes les politesses possibles", ordonne le chant d'un Te Deum à l'occasion du mariage de Georges III et légitime ainsi sa décision: "Le Dieu des armées qui dispose à son gré des couronnes, et qui étend ou restreint selon son bon plaisir les limites des empires, nous ayant fait passer selơn ses décrets éternels sous la domination de Sa Majesté Britannique, il est de notre devoir, fondé sur la loi naturelle même, de nous intéresser à tout ce qui peut la regarder"'32. Le vicaire général de Québec écrivait l'histoire à la manière de l'auteur du Discours sur l'histoire universelle et semble avoir lu l'oraison funèbre d'Henriette de France. C'est dans ce mandement que M. Briand prescrit de prier pour le roi de Grande-Bretagne au canon de la messe. A ceux qui protestèrent contre cette initiative, il répondit que les Anglais "sont nos maîtres; et nous leur devons ce que nous devions aux Français lorsqu'ils l'étaient"

29. Murray a Amherst, [ ?] novembre 1759, Papiers Murray, APC, M. 898 А.

30. "Mandement pour faire chanter un Te Deum dans toutes les paroisses du Gouvernement de Montréal a l'occasion du couronnement et du mariage de $\mathbf{S a}$ Majesté le roi George III", ler février 1762, Mandements, 2: 157.

31. "Lettre circulaire a $M M$ les Curés qui seront dans les quartiers où il est à craindre que l'ennemi ne pénètre", 5 juin 1759, Mandements, 2: 139.

32. "Mandement pour faire chanter un $T e$ Deum en action de grâces du mariage du roi George III", 4 février 1762, ibid., 2: 160.

33. M. J.-O. Briand, vicaire général, au grand vicaire Montgolfier, corrępondance de Mgr Jean Olivier Briand, [1762 ? ], Rapport de l'archiviste de la province de Québec pour 1929-1980 (RAPQ), 50. 
Parmi les dirigeants ecclésiastiques, M. Perrault, grand vicaire aux Trois-Rivières, apparaît avoir été le seul qui ait pressenti les conséquences d'une conquête anglaise pour l'Eglise canadienne. L'une de ses lettres au chanoine Briand laisse deviner ses inquiétudes: "Nous aurons de grands arrangements à prendre, bien des croix et des humiliations à essuyer, supposé que le pays reste à l'Angleterre, pour y soutenir la religion"34. Ayant appris que l'Espagne s'était alliée à la France, le chanoine Perrault confie à son collègue de Québec que "la continuation de la guerre nous la [la paix ] procurera un peu plus conforme à vos désirs, aux miens et à ceux des bons patriotes de ce pays" 35 .

Les "bons patriotes" ne semblent pas avoir été très nombreux. La majorité se pliait aux événements avec une bonne grâce remarquable. La vie continuait. L'occupant avait réussi à rassurer pleinement la population.

\section{Devant le fait accompli}

Les mandements du clergé et les adresses des habitants à l'occasion de la ratification du traité de paix sont très révélateurs.

Le grand vicaire des Trois-Rivières croit que la "paix est faite, Nos Très Chers Frères, pour le bonheur de l'humanité". Il prévoit que le Canada "reste pour jamais au glorieux vainqueur George III, Roi de la Grande-Bretagne". Un éloge du souverain et un appel à la reconnaissance, à la fidélité, à la "soumission parfaite" que lui doivent ses sujets canadiens complètent le document. M. Perrault affirme même que la religion dépend de "cette fidélite" et de "cette soumission parfaite" ${ }^{36}$.

M. Briand fait l'histoire du régime militaire dont la douceur a démontré la miséricorde de Dieu à l'égard des vaincus. Sous le nouveau gouvernement, le peuple goatera "les douceurs d'une paix heureuse et durable". Le vicaire général insiste sur le loyalisme monarchique: "Soyez exacts à remplir les devoirs de sujets fidèles et atta-

34. Perrault a Briand, fin mars 1762, dans Marcel Trudel, Le Régime militaire dans le Gouvernemont des Trois-Rivières, 1760-1764 (Trois-Rivières, 1952), 134.

35. Perrault a Briand, 12 avril 1762, dans $i b i d .$, 142-143.

36. "Mandement ordonnant un Te Deum en action de grâces de la paix", 26 mai 1763, Mandements, 2: 167-168. 
chés à leur prince; et vous aurez la consolation de trouver un roi débonnaire, bienveillant et appliqué à vous rendre heureux, et favorable à votre religion... rien ne peut vous dispenser d'une parfaite obéissance, d'une scrupuleuse et exacte fidélité, et d'un inviolable et sincère attachement à votre nouveau Monarque et aux intérêts de la nation à laquelle nous venons d'être agrégés"37.

M. Montgolfier tient le même langage. Remémorant les bontés du nouveau souverain, il s'accommode assez facilement du changement d'empire: "Vous goûtez déjà depuis plusieurs années les douceurs de son règne". Il insiste sur le fait que par la paix "vos biens spirituels et temporels y sont également ménagés ${ }^{38}$."

Fidèles et pasteurs chantèrent des Te Deum d'action de grâces. Nous ne saurons jamais combien éprouvèrent les sentiments de ce brave curé qui avouera à son supérieur: "J'ai chanté le Te Deum, selon votre mandement, oculis lacrymantibus" ${ }^{39}$. Il semble, cependant, que la majorité accepta assez facilement la conquête. On fit face à la fatalité avec un certain fatalisme. Haldimand avait prévu que "le clergé aura de la peine à digérer cette nouvelle [l'annonce de la fin des hostilités ]; mais je me persuade que la plupart des autres habitants se consoleront facilement du changement auquel elle les prépare" ${ }^{40}$. Madame d'Youville, sans cacher son inquiétude au point de vue matériel, se rassure: "Si nous sommes aussi libres d'exercer notre religion et de faire tout le bien que nous trouvons a faire comme nous l'avons été depuis que nous sommes sous la domination anglaise, nous ne serons pas à plaindre pour le spirituel, mais pour le temporel il $\mathrm{y}$ aura plus de misères; on ne trouve pas a gagner sa vie avec eux [ les Anglais] comme avec les Français, mais j'espère que la Providence y suppléera ${ }^{41}$."

37. "Mandement pour faire chanter un Te Deum en action de grâces pour le bienfait de la paix," 4 juin 1763, ibid., 2: 169-170.

38. "Mandement de Monsieur le Vicaire Général du diocèse de Québec pour faire chanter un Te Deum en action de grâce de la paix", 28 juillet 1763, ibid., 2 : 171, 172.

39. Récollet Théodore, curé de Saint-Joseph de Beauce, a Briand, 22 juin 1763, dans Trudel, Régime militaire, 143-144. 1: 266.

40. Haldimand à Amherst, 12 février 1763, Collection Haldimand, APC, B

41. Madame d'Youville a Villard, 5 aout 1763, dans A. Ferland-Angers, Mere d'Youville, première fondatrice canadienne (Montréal, 1945), 215. 
Les bourgeois de Québec prophétisent qu'ils seront "agrégés sans retour au corps des sujets de la couronne d'Angleterre" "2. Ils y voient un décret de l'Etre Suprême: "C'est à nous de nous y conformer, et d'être aussi fidèles sujets de notre nouveau monarque, que nous l'avons été, ou dû être, du Roi de France. Hé, comment ne le serionsnous pas, après avoir éprouvé, en qualité de sujets vaincus, de la manière la plus marquée, la douceur, la justice et la modération de son gouvernement, après la bonté paternelle qu'il a eue pour nous... en nous ayant traités comme ses anciens sujets? Que n'avons-nous donc pas lieu d'espérer actuellement?"' ${ }_{3}$ Les bourgeois de Québec ne manquaient pas d'enthousiasme ni d'optimisme.

\section{Conclusion}

Devons-nous être surpris de constater que la majorité accepta son sort sans protester? Il semble même que plusieurs Canadiens, que l'on peut juger coupables d'inconscience, se réjouirent du "changement de domination". Craignant le pire, les vaincus découvrirent, non sans étonnement, que le conquérant les traitait avec bienveillance. L'excès de peur qu'ils avaient éprouvé explique, en bonne partie, leur soumission presque spontanée, au début de l'occupation anglaise. Comparant la situation à celle qui prévalait avant la conquête, ils se jugèrent, sinon privilégiés, du moins très chanceux. L'historien anglo-canadien Arthur R.M. Lower affirme: "Si les Français du Canada avaient eu la liberté de choisir leurs conquérants, ils n'auraient pas pu faire un choix meilleur que celui que le sort avait fait pour eux ${ }^{44}$ '. Pour compléter la pensée de M. Lower, reconnaissons que le conquérant pouvait difficilement trouver des conquis mieux disposés.

La génération de 1760 vivait à l'époque du despotisme éclairé. Ces hommes et ces femmes d'ancien régime ne connaissaient qu'un

42. L'expression "agrége" semble avoir fait fortune. Le chanoine Briand l'emploie dans son mandement du 4 juin 1763, voir supra. On trouve la même expression dans une lettre de Philippe de Rocheblave a Carleton [ ? ], 4 juillet 1778, Collection Haldimand, APC, B 122: 98.

43. "Adresse des bourgeois de Québec à l'occasion du traité de paix", 4 juin 1763, dans Gosselin, Eglise après la conquête, 1: 59-60. 64.

44. Arthur R.M. Lower, Colony to Nation: A History of Canada (Toronto, 1946) 
seul système politique: la monarchie absolue. Ils l'acceptaient tout naturellement, sans discussion. La Révolution américaine et la Révolution française n'avaient pas encore prêché le droit des peuples à disposer d'eux-mêmes. Le nationalisme moderne n'existait pas. Monarchistes dans l'âme ${ }^{45}$, habitués à vivre dans une société fortement hiérarchisée ${ }^{46}$, attachés à leur coin de terre, les Canadiens accueillirent leurs nouveaux maîtres sans méfiance. Pouvaient-ils prévoir que la conquête anglaise modifierait fatalement et radicalement leur évolution historique? Ils se savaient l'immense majorité au Canada. Tout naturellement, ils avaient l'illusion qu'il en serait toujours ainsi. Plusieurs gouvernants anglais ne pensaient pas différemment". Même "agrégés" à une nation étrangère et membres d'un nouvel empire, ils se croyaient en sécurité sous la tutelle d'un roi "débonnaire, bienveillant et appliqué à vous rendre heureux". Dans ces conditions, pourquoi se seraient-ils inquiétés?

Pour les Canadiens de 1760 , la conquête se ramenait à un simple changement dans la succession au trône. Louis XV avait abandonné la colonie. L'ancien roi était mort. Les nouveaux sujets de Sa Majesté Britannique se montraient prêts à crier: "Vive le roi Georges".

Professeur agrege,

Institut d'histoire,

Faculte des Lettres,

Universite de Montreal.

Michel Brunet

45. Voir chapitre "Sous le signe de l'absolutisme", dans Frégault, La Civilisation de la Nouvella-France (Montréal, 1944), 121-167.

46. Murray a Halifax, 15 octobre 1764, APC, Q 2: 206.

47. Carleton au Secrétaire d'Etat, 25 novembre 1767, dans Histoire du Canada par les textes, 114. 